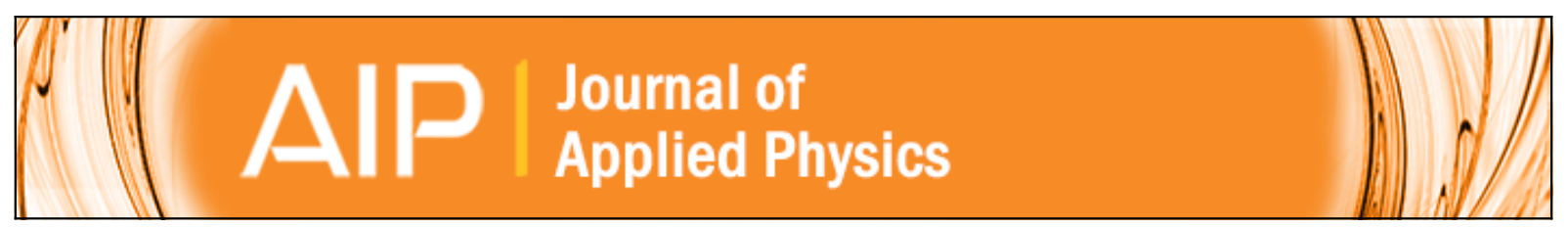

\title{
Surface characterization of InP trenches embedded in oxide using scanning probe microscopy
}

Manuel Mannarino, Ravi Chintala, Alain Moussa, Clement Merckling, Pierre Eyben, Kristof Paredis, and Wilfried

Vandervorst

Citation: Journal of Applied Physics 118, 225304 (2015); doi: 10.1063/1.4936895

View online: http://dx.doi.org/10.1063/1.4936895

View Table of Contents: http://scitation.aip.org/content/aip/journal/jap/118/22?ver=pdfcov

Published by the AIP Publishing

\section{Articles you may be interested in}

Preparation and atomic structure of reconstructed (0001) InGaN surfaces

J. Appl. Phys. 112, 033509 (2012); 10.1063/1.4743000

InGaAs surface preparation for atomic layer deposition by hydrogen cleaning and improvement with high temperature anneal

J. Appl. Phys. 110, 013713 (2011); 10.1063/1.3597791

Real-time scanning tunneling microscopy observations of the oxidation of a TiPt(111)-(2×2) surface alloy using $\mathrm{O} 2$ and $\mathrm{NO} 2 \mathrm{a}$ )

J. Vac. Sci. Technol. A 26, 1336 (2008); 10.1116/1.2969903

The role of the InGaAs surface in selective area epitaxy of quantum dots by indium segregation

Appl. Phys. Lett. 84, 3031 (2004); 10.1063/1.1705731

Reflection high-energy electron diffraction and scanning tunneling microscopy study of $\ln P(001)$ surface reconstructions

J. Vac. Sci. Technol. A 18, 1492 (2000); 10.1116/1.582373

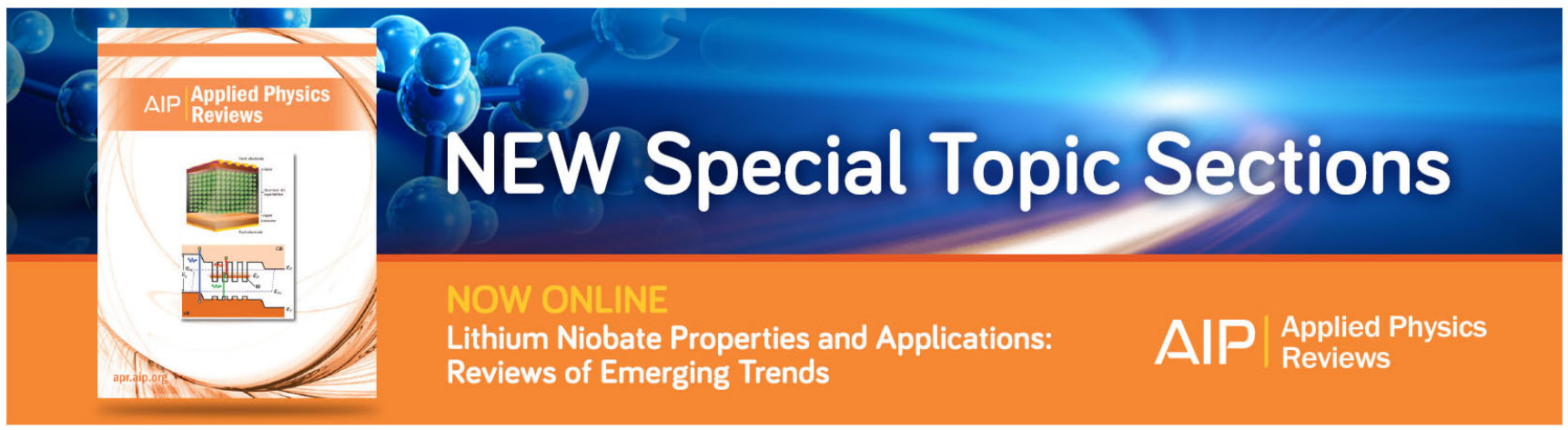




\title{
Surface characterization of InP trenches embedded in oxide using scanning probe microscopy
}

\author{
Manuel Mannarino, ${ }^{1,2, a)}$ Ravi Chintala, ${ }^{1,2}$ Alain Moussa, ${ }^{1}$ Clement Merckling, ${ }^{1}$ \\ Pierre Eyben, ${ }^{1}$ Kristof Paredis, ${ }^{1}$ and Wilfried Vandervorst ${ }^{1,2}$ \\ ${ }^{1}$ IMEC, Kapeldreef 75, B-3001 Heverlee, Belgium \\ ${ }^{2}$ Department of Physics and Astronomy (IKS), KU Leuven, Celestijnenlaan 200D, B-3001 Heverlee (Leuven), \\ Belgium
}

(Received 8 September 2015; accepted 14 November 2015; published online 8 December 2015)

\begin{abstract}
Metrology for structural and electrical analyses at device level has been identified as one of the major challenges to be resolved for the sub- $14 \mathrm{~nm}$ technology nodes. In these advanced nodes, new high mobility semiconductors, such as III-V compounds, are grown in narrow trenches on a Si substrate. Probing the nature of the defects, the defect density, and the role of processing steps on the surface of such structures are prime metrology requirements. In order to enable defect analysis on a (III-V) surface, a proper sample preparation for oxide removal is of primary importance. In this work, the effectiveness of different chemical cleanings and thermal annealing procedures is investigated on both blanket InP and oxide embedded InP trenches by means of scanning probe microscopy techniques. It is found that the most effective approach is a combination of an $\mathrm{HCl}$-based chemical cleaning combined with a low-temperature thermal annealing leading to an oxide free surface with atomically flat areas. Scanning tunneling microscopy (STM) has been the preferred method for such investigations on blanket films due to its intrinsic sub-nm spatial resolution. However, its application on oxide embedded structures is non-trivial. To perform STM on the trenches of interest (generally $<20 \mathrm{~nm}$ wide), we propose a combination of non-contact atomic force microscopy and STM using the same conductive atomic force microscopy tip Our results prove that with these procedures, it is possible to perform STM in narrow InP trenches showing stacking faults and surface reconstruction. Significant differences in terms of roughness and terrace formation are also observed between the blanket and the oxide embedded InP. (C) 2015 AIP Publishing LLC. [http://dx.doi.org/10.1063/1.4936895]
\end{abstract}

\section{INTRODUCTION}

With the continuous downscaling within the nanoelectronics industry, standard complementary metal-oxide semiconductor (CMOS) structures and Si based devices are facing significant limitations in terms of speed, power dissipation, and current leakage. ${ }^{1}$ In an attempt to overcome these limitations, one solution being explored is through replacing the $\mathrm{Si}$ channel used in CMOS devices with high carrier mobility materials such as III-V compounds. However, for technical, mechanical, and economic reasons, the main carrier must remain a $\mathrm{Si}$-wafer. ${ }^{1}$ The growth of III-V materials onto a $\mathrm{Si}$ substrate leads to the formation of crystallographic defects and anti-phase boundaries (APBs). The former are mostly generated by stress relaxation induced by the lattice mismatch, while the latter occur because of a combination of single atomic steps on the Si surface and the polar nature of III-V materials. ${ }^{2}$ It has been shown that the crystallographic defect density can be reduced by growing the III-V material within narrow trenches with an aspect ratio greater than two, thereby confining the defects to the side wall, i.e., aspect-ratio trapping (ART). ${ }^{3}$ Even with ART, one still depends on a buffer layer, commonly InP, to ultimately achieve a defect free high mobility channel layer. When properly engineered,

\footnotetext{
a) Author to whom correspondence should be addressed. Electronic addresses: manuel.mannarino@imec.be and manuelmannarino@gmail.com
}

one would expect to find a higher defect density at the bottom of the buffer layer and at the top a defect free material. ${ }^{4}$ On the other hand, APBs can be limited by introducing a Ge layer to control the double atomic step density at the buffer layer interface $^{5}$ or by growing the III-V buffer layer on a V-shaped Si substrate with $\{111\}$ facets. ${ }^{6}$ A typical III-V nMOS heterostructure is shown in Fig. 1(a). Note that the whole structure is surrounded by oxide (shallow trench isolation-STI). A barrier layer, typically $\operatorname{In}_{\mathrm{x}} \mathrm{Al}_{1-\mathrm{x}} \mathrm{As}$, is placed above the ARTbased InP (cf. Fig. 1(b)). This introduces a higher conduction
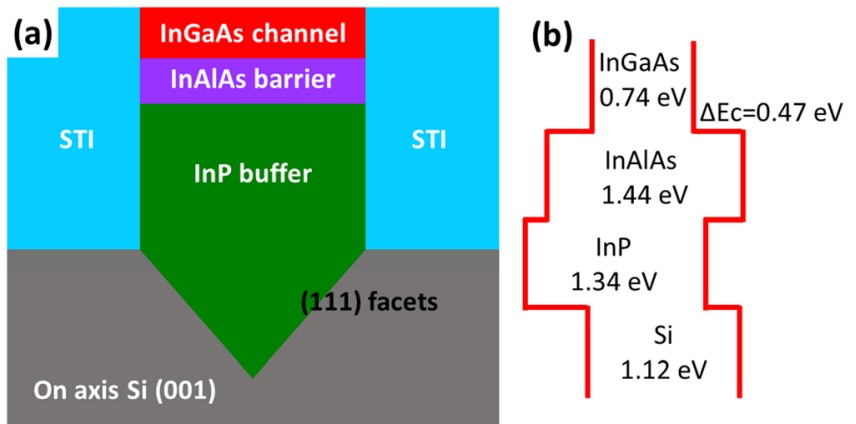

FIG. 1. (a) Typical hetero-structure for III-V nMOS applications on a Si substrate. The buffer layer (InP) is used to compensate the lattice mismatch between $\mathrm{Si}$ and the channel layer (InGaAs), whereas the barrier layer (InAlAs) limits the leakage current. The channel layer is grown on top of the barrier. (b) Band diagram of the different layers considering $\operatorname{In}_{0.53} \mathrm{Ga}_{0.47} \mathrm{As}$ and $\mathrm{In}_{0.52} \mathrm{Al}_{0.48} \mathrm{As}$. 
band offset between the high mobility channel (InGaAs in this case) and the InP buffer layer, thereby reducing any leakage current component present. ${ }^{7}$ Finally, on top of the barrier layer a high mobility $\operatorname{In}_{x} \mathrm{Ga}_{1-x}$ As channel is grown. The overall electrical properties of the channel are strongly influenced by the quality of the underlying layers. Therefore, achieving an InP buffer layer with an almost defect free surface is of primary importance. Besides, the identification and characterization of the planar (stacking faults (SFs), twins, APBs) and linear (dislocations) defects formed during the growth processes are fundamental to the material growth, device formation, and process optimization.

The metrology required for the structural and electrical analysis at a device level today has been identified as one of the major challenges to be resolved by the ITRS roadmap, ${ }^{8}$ especially for the next technology nodes. Hence, developing the metrology to be able to probe the nature of the defects and their electrical behavior within very confined volumes is required. Among the scanning probe microscopy (SPM) based techniques, scanning tunneling microscopy (STM) is well known for its sub-nm spatial resolution for topographic analysis and its ability to evaluate the electrical properties of (semi-)conducting materials over a very localized area., 9,10 Therefore, STM should in principle be suitable for the investigation of the crystalline properties and the presence of any defects in (III-V) semiconductors. However, since STM uses the tunneling current as a feedback signal to control the height of the tip, only conductive surfaces can be probed. This means that when STM has to be performed on a small device surrounded by oxide, the tip needs to be positioned very precisely above the narrow trench in order to avoid the tip colliding with the oxide. In this work, we overcome the STM-tip positioning by using a combination of non-contact atomic force microscopy (nc-AFM) and STM in subsequent scans. Despite the fact that the combination of nc-AFM and STM with the same tip has already been demonstrated, ${ }^{1-14}$ in most of the existing literature the tip is scanned in constant height mode such that tunneling current and shift of the resonant frequency can be recorded at the same time. Only in very few cases, nc-AFM and STM are performed in subsequent scans by using their respective feedback mechanisms. ${ }^{15}$ An important point to note is that in all previously reported works, the sample surfaces were almost atomically flat. Contrary to this, our material grown in trenches includes steps higher than $10-15 \mathrm{~nm}$ at the interface with the STI. This makes the study of such a structure non-trivial precluding, for instance, the possibility to perform simultaneous ncAFM and STM measurements.

Another challenge arises from the fact that when dealing with structures prepared on a $300 \mathrm{~mm}$ wafer in a standard (industrial) CMOS processing line, structures have undergone (surface roughening) processes like chemical mechanical polishing (CMP) and they are exposed to the (oxidizing) ambient. As such, these surfaces differ significantly from in-situ grown and characterized surfaces. To enable defect characterization at an atomic scale by STM, oxide free and atomically flat surfaces are fundamental requirements. The standard approaches for (native) oxide removal typically involve a high temperature anneal which, in the case of InP, leads to surface degradation due to $\mathrm{P}$ desorption and In nucleation. ${ }^{16,17}$ Wet chemical cleanings for oxide removal are efficient but do not provide a proper surface passivation ${ }^{18-21}$ and cannot prevent some III-V reoxidation during and after the rinsing bath. Finally, for sputtering approaches it is still not clear whether the surface is affected by implantation and/or roughening. ${ }^{22,23}$

The work presented herein focuses on the analysis of epitaxial InP grown on a InP substrate (2-in. wafer) and InP grown on $\mathrm{V}$-shaped Si trenches. ${ }^{24} \mathrm{We}$ have investigated the effectiveness of thermal annealing and chemical cleanings $(\mathrm{HF}$ and $\mathrm{HCl}$ ) for native oxide removal and terrace formation on the blanket samples. Furthermore, we have subsequently explored the combination of nc-AFM and STM, using the same conductive atomic force microscopy (AFM) tip, to successfully analyze the InP embedded in an oxide matrix.

\section{EXPERIMENTAL}

The 2-in. InP (100) blanket wafers adopted for this work were doped with zinc to a carrier concentration of $1 \times 10^{18} \mathrm{~cm}^{-3}$ (wafers provided by AXT Inc.). Patterned samples were produced in house using our standard process as described in more detail within Ref. 24. They are based on the creation of narrow trenches with oxide isolation (STI), epitaxial growth on V-shaped Si trenches, and a final CMP step for the removal of any overgrown material. The samples were subsequently treated ex-situ and under dark conditions (to avoid photo-stimulated etching) in either a $\mathrm{HF}$ $2 \%$ or $0.25 \mathrm{M} \mathrm{H}_{2} \mathrm{O}_{2} / 1 \mathrm{M} \mathrm{HCl}$ and $2 \mathrm{M} \mathrm{HCl}$ solutions. After the chemical treatment, the samples were rinsed in deionized water and exposed to air for about 3-5 min. Once inside the load lock of the UHV chamber, a pressure of $3 \times 10^{-7}$ Torr was reached in $\sim 5 \mathrm{~min}$. To desorb any hydrocarbons on the sample surface, the load lock was baked $\left(160{ }^{\circ} \mathrm{C}\right.$ for $\left.6 \mathrm{~h}\right)$ with the sample inside. After transferring the sample into the UHV-SPM chamber, a further thermal anneal was performed using radiative heating from a $\mathrm{W}$ filament underneath the sample. In this case, the heating rate was initially $5^{\circ} \mathrm{C} / \mathrm{min}$ but was lowered to $4^{\circ} \mathrm{C} / \mathrm{min}$ and $3{ }^{\circ} \mathrm{C} / \mathrm{min}$ reaching $220^{\circ} \mathrm{C}$ and $280^{\circ} \mathrm{C}$, respectively. The cooling process followed the reverse trend. STM and nc-AFM were performed in the UHV system equipped with a scanning electron microscope (RHK 3500 HT). All the measurements were carried out at room temperature under a vacuum of $\sim 5 \times 10^{-10}$ Torr. Commercial Pt/Ir STM tips and Pt/Ir coated AFM tips with a cantilever spring constant of $42 \mathrm{~N} / \mathrm{m}$ (nominal value) were used for the measurements. All the data were analyzed with the WSxM software. ${ }^{25}$

\section{RESULTS AND DISCUSSION}

\section{A. Preparation of an oxide free, undamaged InP surface}

In order to analyze the InP surface at atomic level, the native oxide has to be removed and the surface should ideally exhibit atomically flat regions. Although such an oxide can be removed by a simple thermal anneal, phosphorus evaporation and indium nucleation are phenomena that have been previously observed when InP is raised to a temperature 
suitable for the oxide removal. In many published works, this problem is tackled by annealing the sample under $\mathrm{P}_{2}^{26,27}$ or by $\mathrm{Ar}^{+}$sputtering in combination with a thermal annealing step. ${ }^{28,29}$ As these approaches are not applicable here because they would degrade the material integrity, to avoid topographic and/or compositional changes (e.g., implantation), ${ }^{22,23}$ we only made use of controlled thermal annealing within the UHV chamber. During our experiments, we did observe InP decomposition when we reached $\sim 400^{\circ} \mathrm{C}$. Figure S1 of the supplementary material ${ }^{30}$ shows an optical image of a sample annealed at $400^{\circ} \mathrm{C}$. Different morphological shapes of the indium metallic clusters are seen to co-exist on the same sample, i.e., $\langle 011\rangle$ oriented, merged, and randomly distributed. As the change from one shape to another requires only a few tens of ${ }^{\circ} \mathrm{C},{ }^{16}$ their co-existence is not surprising because it is believed to be driven by their formation kinetics. For example, by keeping a constant temperature of $375^{\circ} \mathrm{C}$, the surface starts to degrade after $\sim 15 \mathrm{~min}$, whereas at $\sim 400^{\circ} \mathrm{C}$, the degradation is immediate. By optimizing the annealing temperature and the heating rate, it is therefore possible to remove the oxide layer and to limit, up to a certain extent, the surface degradation. As one might expect, at $375^{\circ} \mathrm{C}$ it is much easier to limit the morphological changes compared to $400^{\circ} \mathrm{C}$. Figure 2(a) shows an STM analysis done on an InP sample annealed at $375^{\circ} \mathrm{C}$ for $15 \mathrm{~min}$. Two atomically flat terraces are identified. The surface consists of a $4 \times 2$ reconstruction with a distance of $\sim 1.6 \mathrm{~nm}$ between the atomic rows along the [011] direction. The $4 \times 2$ reconstruction is also an In-rich surface, ${ }^{31,32}$ in agreement with that expected since phosphorus is evaporating during the annealing process. The height of the measured steps is $\sim 0.85 \mathrm{~nm}$ corresponding to 3 atomic steps $(0.293 \mathrm{~nm})$ and therefore it may be created by the movement of an In droplet on the surface. Since such a droplet creates tracks and at the same time releases some material during its movement (cf. Figs. 2(c), 2(d), and S1 of the supplementary material ${ }^{30}$ ), the small visible protrusion observed in Fig. 2(a) may be linked to an In-cluster. Very straight $\langle 011\rangle$ oriented terraces, characteristic of a moving droplet, are also observed on the same sample (cf. Fig. S2 of the supplementary material ${ }^{30}$ ). Further analysis of the same sample with a larger field of view such as using an optical microscope and tapping mode AFM clearly shows droplets of $1-2 \mu \mathrm{m}$ in size as well as clean areas up to $30 \mu \mathrm{m}$ in length. From the optical image of Fig. 2(c), we identify two different mechanisms of indium nucleation. One is a "walking" process whereby the particle collects material by moving over the surface leaving a track behind due to the removed material. The other is an "agglomeration" process whereby the particle collects material from the surrounding area creating a depleted zone around it. The latter process is easily identifiable in the tapping AFM image of Fig. 2(d) where a quantitative evaluation of the particle formation is obtained. The height of the particles is a few hundred nm, while their geometry appears independent of the nucleation mechanism following the crystallographic orientation. However for the moving particles, the edges are clearly seen to be rounded. The track created by the move of the droplet and the depression created by the standing one are both $\sim 5-10 \mathrm{~nm}$ in depth.

The results presented thus far show that by thermal annealing alone, it is not possible to remove the oxide layer without generating surface damage. Moreover, lowering the temperature does not lead to an oxide-free surface. Targeting InP native oxide removal from the sample surface, we have explored different chemical cleanings. Among the several recipes available in the literature, we focused on $\mathrm{HF}$ and $\mathrm{HCl}$ based cleanings because they have been shown to provide the best results in terms of surface morphology and passivation. HF removes the native oxide ${ }^{19}$ while at the same time it has no etching effect on the InP. Therefore, no surface morphological changes are expected. $\mathrm{HCl}$ has a smoothening effect in combination with a distinct terrace formation. ${ }^{36}$ Therefore, large $(>100 \mathrm{~nm})$ atomically flat areas are expected after the $\mathrm{HCl}$ dip. Although $\mathrm{H}_{2} \mathrm{SO}_{4}$ has been shown to act in a similar way to $\mathrm{HCl}^{36}$ it was not adopted here because the chemisorbed sulfur cannot be completely removed at a temperature lower than the decomposition temperature of InP. ${ }^{33}$

HF cleaning partially terminates the surface with fluorine. ${ }^{21}$ Since such a surface is hydrophilic and re-oxidizes when rinsed in water and/or exposed to air, an annealing step was required. Fluorine is known to desorb at $\sim 230{ }^{\circ} \mathrm{C}^{21}$ but
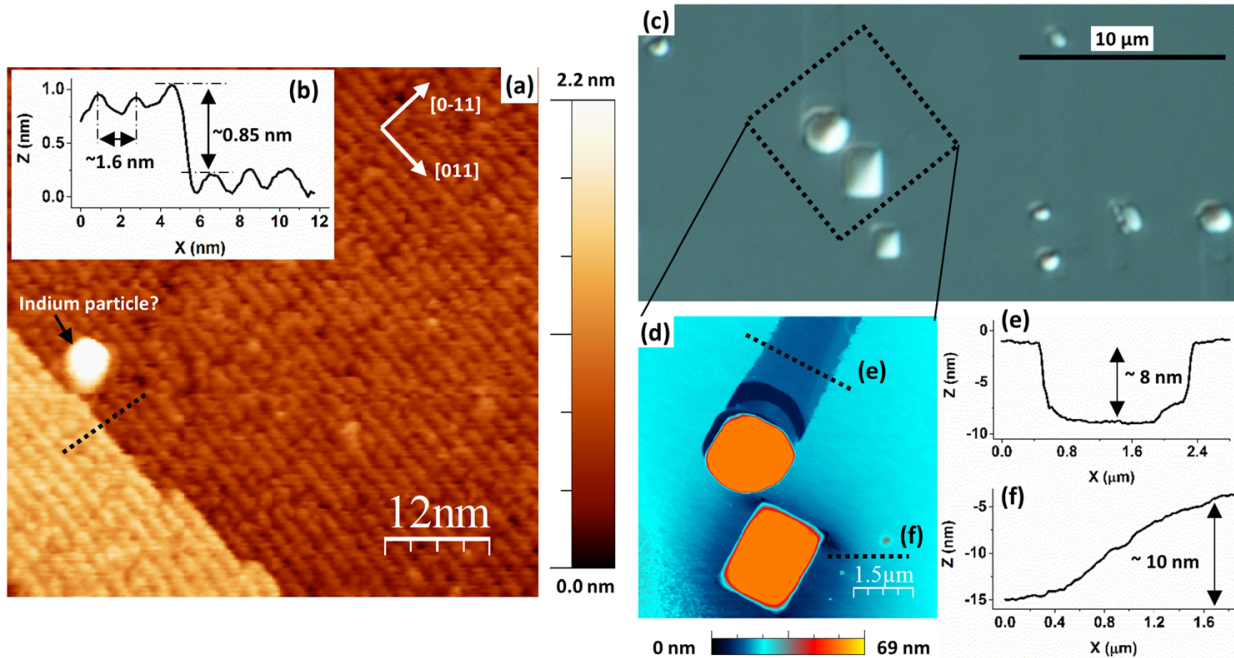

FIG. 2. (a) STM on an InP blanket wafer after thermal annealing at $375^{\circ} \mathrm{C}$. Two atomically flat terraces are visible. The measured step between them is $\sim 0.85 \mathrm{~nm}$. The surface consists of a $4 \times 2$ reconstruction with a distance of $\sim 1.6 \mathrm{~nm}$ between the atomic rows along the [011] direction. The bright spot probably reflects an indium droplet. Scan area $=60 \times 60 \mathrm{~nm}^{2} ; \mathrm{V}_{\mathrm{s}}=3.00 \mathrm{~V}$; $I_{\text {set }}=160 \mathrm{pA}$. (b) The inset shows a cross section line taken across the two terraces. (c) Optical image of the InP surface where two different mechanisms of indium nucleation are visible. (d) Topographic analysis of the highlighted area by tapping AFM. Cross section lines taken in the AFM image show the depth (e) of the track and (f) of the depleted area. 


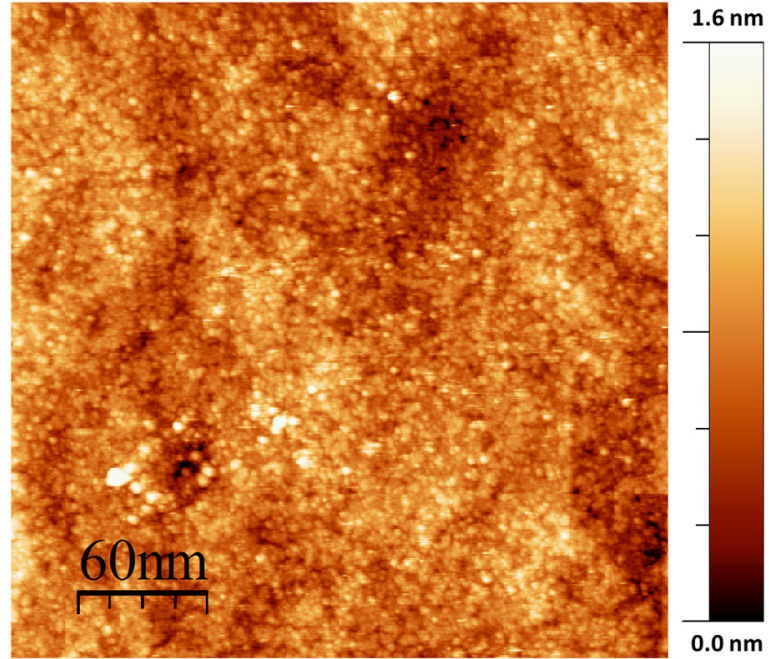

FIG. 3. STM on InP after HF dip and thermal annealing at $340^{\circ} \mathrm{C}$. No surface reconstruction nor atomic terraces are visible. Scan area $=300$ $\times 300 \mathrm{~nm}^{2}, \mathrm{~V}_{\mathrm{s}}=3.00 \mathrm{~V}, \mathrm{I}_{\mathrm{set}}=100 \mathrm{pA}$.

to remove the oxide a higher temperature is required. Therefore, after a dip in a $2 \%$ aqueous $\mathrm{HF}$ solution for $1.5 \mathrm{~min}$, the sample was annealed at $340^{\circ} \mathrm{C}$ within the UHV chamber. An STM image of the sample surface is shown in Fig. 3. Although we can evaluate the morphological quality of the surface, it was not possible to identify atomic-scale crystallographic defects because no distinguishable atomically flat terraces are observed. Additionally, no surface reconstruction can be observed even in small scan areas. An InP (001) oxide-free surface implies the presence of dangling bonds and a rearrangement of the atoms on the surface in order to minimize their surface energy. However, a regular and ordered surface, as observed for instance in Fig. 2(a), is only possible within atomically flat areas. It should be noted that the investigated sample did undergo a CMP step which gives rise to surface roughness which cannot be recovered by our current HF cleaning and annealing processes. A higher temperature thermal anneal could provide enough energy to induce an atomic rearrangement of the top surface. However, this would probably lead, as in the case of the simple thermal annealing, to a degradation of the surface.

The second recipe investigated in this work is based on $\mathrm{H}_{2} \mathrm{O}_{2}$ and $\mathrm{HCl}$. It consists of an etching step and an oxide removal step. ${ }^{34,35}$ In particular, for the etching we used $0.25 \mathrm{M}$ $\mathrm{H}_{2} \mathrm{O}_{2} / 1 \mathrm{M} \mathrm{HCl}$ for $5 \mathrm{~min}$, while the oxide removal was carried out using $2 \mathrm{M} \mathrm{HCl}$ for $5 \mathrm{~min}$. During the etching process, a few nanometers of the top surface are removed by an oxide formation/oxide dissolution process. ${ }^{34}$ In this work, we are mostly interested in planar and linear defects arising from the lower region of the buffer layer which form during growth. Therefore, even if a few nanometers are removed from the top surface, the defects and their distribution are not affected. Through the second step, the $\mathrm{HCl}$ dip, the oxide layer is removed producing a partially passivated surface. $^{18,21}$ This cleaning process allows to achieve very smooth surfaces with distinct atomically flat terraces. ${ }^{34-36}$ Figure 4(a) shows the STM analysis of the InP surface after the cleaning and the insertion into the UHV chamber. Elongated terraces up to $\sim 100 \mathrm{~nm}$ in length are observed. As atoms are less stable at the step edges, they are removed during the $\mathrm{HCl}$ dip. Therefore, atomically flat terraces are generated and enlarged. The step height between the terraces is $\sim 0.3 \mathrm{~nm}$ (single atomic step). However, when reducing the scan area and focusing on one single atomic flat terrace, the images became significantly noisy and blurry (not shown). This is due to the partial surface passivation combined with a thin oxide layer formed during the sample mounting. ${ }^{18,21}$ In order to obtain a completely clean surface, this chemical treatment was combined with a thermal annealing inside the UHV chamber. After annealing at $300{ }^{\circ} \mathrm{C}$, an oxide-free and reconstructed $(4 \times 2)$ InP surface was obtained, as shown in Fig. 4(c). The required temperature was $\sim 70^{\circ} \mathrm{C}$ lower than

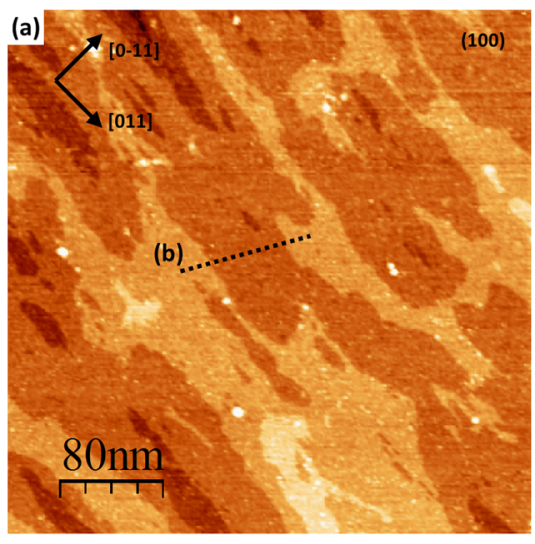

(b)

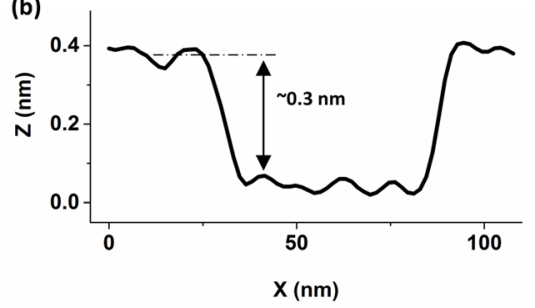

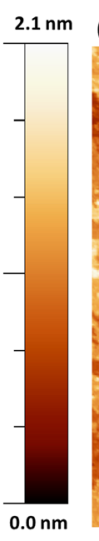
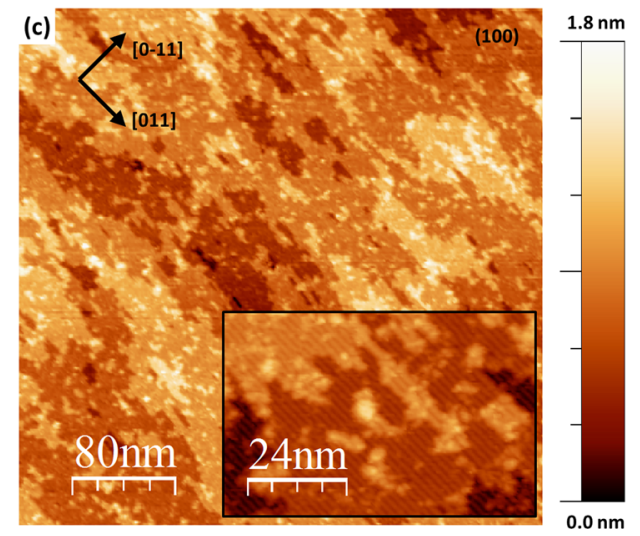

(d)

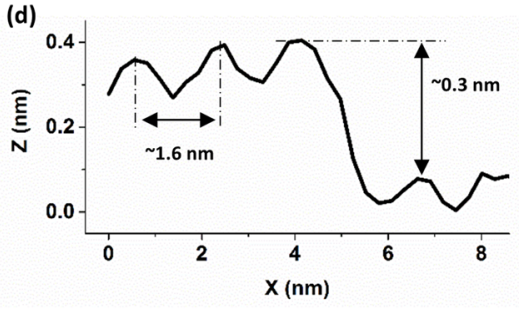

FIG. 4. (a) STM on InP (100) blanket after an $\mathrm{HCl}$-based wet cleaning. Atomic terraces in the order of $100 \mathrm{~nm}$ long are generated during the $\mathrm{HCl}$ dip. Scan area $=400 \times 400 \mathrm{~nm}^{2}, \mathrm{~V}_{\mathrm{s}}=2.80 \mathrm{~V}, \mathrm{I}_{\mathrm{set}}$ $=150 \mathrm{pA}$. (b) A cross section line taken across the terraces shows a step height of $\sim 0.3 \mathrm{~nm}$. (c) STM after wet cleaning and thermal annealing at $300^{\circ} \mathrm{C}$. Terrace borders show a different morphology compared to the non-annealed sample. Scan area $=400 \times 400 \mathrm{~nm}^{2}, \mathrm{~V}_{\mathrm{s}}$ $=2.80 \mathrm{~V}, \mathrm{I}_{\mathrm{set}}=150 \mathrm{pA}$. In the inset a smaller scan area shows a $4 \times 2$ reconstructed surface. (d) A cross section line taken across two terraces in the inset of (c), an atomic step and the reconstruction profile are identifiable. 
that without chemical treatment which has the added advantage of limiting the surface from degrading. Moreover, considering that all the steps of the InP growth are generally done at temperatures higher than $400{ }^{\circ} \mathrm{C},{ }^{24}$ it minimizes any effect on the original crystallographic defect distribution. The lower thermal budget to achieve oxide evaporation is because the regrown oxide is only $0.2-0.3 \mathrm{~nm}$ as shown by van Dorp et al., ${ }^{35}$ which is substantially thinner than the native oxide. Another feature of interest after thermal annealing is the shape of the edges of the terraces. Compared to the non-annealed sample, where the borders are straight over long distances, here they appear jagged while the terrace size is smaller. This can be explained because atoms at the step edges are chemically less stable; thus, they evaporate and/or move more easily due to the energy acquired during the thermal treatment. This process therefore reduces the terrace size and induces a more random orientation. However, the most important result of Fig. 4(c) is that the surface quality achieved is suitable for STM characterization.

\section{B. STM measurements in narrow trenches embedded in oxides}

To targeting high quality material for the device fabrication, the III-Vs are grown in trenches with widths below $20 \mathrm{~nm}$. Classical measurement techniques must therefore be modified to perform a proper characterization when dealing with such small dimensions. When dealing with relatively large trenches $(>300-500 \mathrm{~nm})$, the STM tip can readily be positioned above the trench by guiding it with a scanning electron microscopy (SEM) image generated simultaneously. This allows to land the STM tip exactly above the InP. However, for smaller dimensions, the resolution limits of the SEM and the physical dimensions of the STM tip require an alternative approach. The approach we have adopted is based on the combination of nc-AFM and STM while using the same conductive AFM probe. The tip is first utilized in ncAFM mode to locate the narrow trench of interest and, once in place, its operation mode is switched to STM (cf. Fig. S3 of the supplementary material ${ }^{30}$ ). In principle, this approach should not have any limitation with respect to the downscaling of the trench. A shifted probing point could only be linked to the different tip-sample interaction area of the two modes. Considering a typical tip radius $<30 \mathrm{~nm}$, a shift larger than a few nanometers seems unlikely. One of the key challenges in this approach is to match the tip and cantilever requirements for the nc-AFM, which uses the cantilever as a force sensor in oscillation mode, to that of the STM which relies on the tunneling current to control the tip height operating in non-oscillation mode. First and foremost the tip must be sharp and have a metallic behavior to reach atomic resolution and enable extraction of electrical information such as I-V spectroscopy. Moreover, a certain cantilever stiffness to avoid snap-in due to the electrostatic forces and low noise in the $\mathrm{z}$-direction is also a requirement of this approach. According to the simulations done by Ozcan and Sitti, ${ }^{37}$ an AFM-cantilever must have a minimum spring constant of $15 \mathrm{~N} / \mathrm{m}$ for use in STM-mode, although this is just

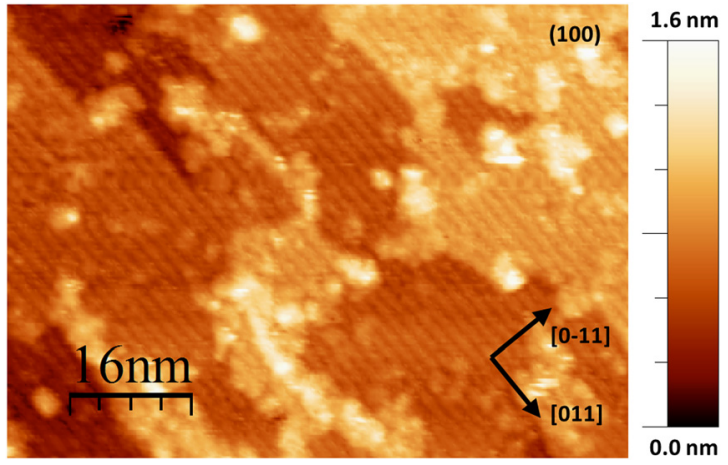

FIG. 5. Surface reconstruction imaged using STM with a Pt/Ir coated AFM tip $42 \mathrm{~N} / \mathrm{m}$ on an $\operatorname{InP}(100)$ blanket sample. Scan area $=80 \times 60 \mathrm{~nm}^{2}$, $\mathrm{V}_{\mathrm{s}}=-2.8 \mathrm{~V}, \mathrm{I}_{\mathrm{set}}=-100 \mathrm{pA}$.

an indicative value since tip radius/shape and bias affect the electrostatic forces. We have screened various tips and the best results are obtained using relatively stiff $\mathrm{Pt} / \mathrm{Ir}$ coated tips with a nominal spring constant of $42 \mathrm{~N} / \mathrm{m}$. Effective stiffness was evaluated by thermal tune ${ }^{38}$ and was found to be $29 \pm 3 \mathrm{~N} / \mathrm{m}$. Figure 5 shows an STM analysis performed on a blanket InP sample using this tip and exhibiting atomic corrugation.

Based on these results we tested the proposed localization approach on a $500 \mathrm{~nm}$ wide InP trench. Figure 6(a) shows a topography image using the nc-AFM mode before switching to STM. As the sample was treated with HF, the InP-trench material is easily identified. Since the HF not only attacks the native oxide on the InP but also etches the surrounding oxide, the trench emerges as a small hillock with respect to the STI. The STI is $\sim 12 \mathrm{~nm}$ lower than InP. Comparing the original surface topography (not shown), where some material removal $(\sim 13 \mathrm{~nm})$ in the trench region is observed after the CMP-step, the vertical etch of the STI was determined to be $\sim 25 \mathrm{~nm}$. The protrusions at the edges of the InP originate from the CMP process which has a lower removal rate at the interface with the STI. Once the trench is centered within the scan area, it is possible to switch to the

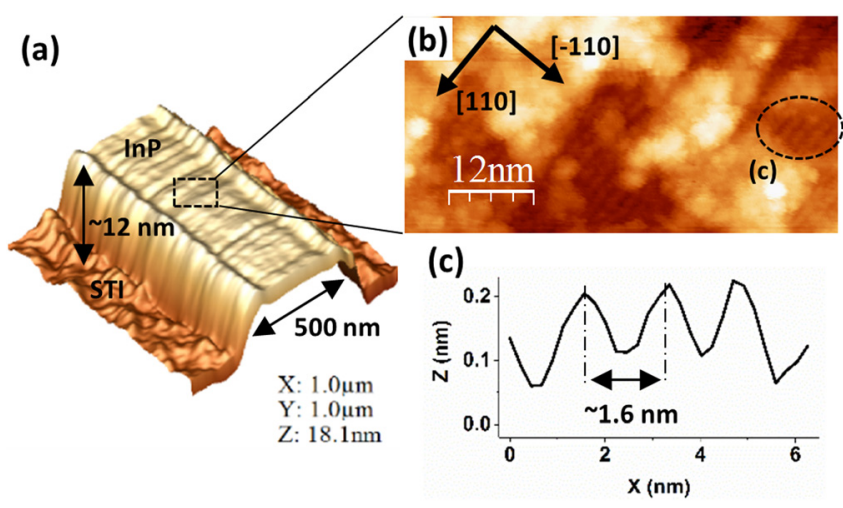

FIG. 6. (a) nc-AFM on a patterned InP sample after HF dip and thermal annealing at $370^{\circ} \mathrm{C}$. The well-shaped InP with the (110) sidewalls is visible. The STI is $\sim 12 \mathrm{~nm}$ lower than the InP. Scan area $=1 \times 1 \mu \mathrm{m}^{2}, \Delta \mathrm{f}=-2 \mathrm{~Hz}$. (b) STM on top of the InP by using the same AFM tip. The surface appears rough but with small atomically flat areas. Locally the small terraces show a $4 \times 2$ surface reconstruction. Scan area $=60 \times 30 \mathrm{~nm}^{2}, \mathrm{~V}_{\mathrm{s}}=-3.00 \mathrm{~V}$, $\mathrm{I}_{\mathrm{set}}=-130 \mathrm{pA}, \mathrm{z}$-scale $=1.4 \mathrm{~nm}$. (c) A cross section line taken in the highlighted area shows a distance of $1.6 \mathrm{~nm}$ between the atomic rows. 
STM-mode. Figure 6(b) shows the STM image of the top InP surface. Compared to the blanket sample, a rougher surface with smaller atomically flat areas is visible. Nevertheless, the terraces are still observed and have a $4 \times 2$ surface reconstruction. The surface reconstruction implies that the oxide layer is completely removed, while the surrounding protrusions can be linked to an initial degradation process activated by the thermal annealing. In this specific case, the sample was annealed at $370^{\circ} \mathrm{C}$ for $10 \mathrm{~min}$. The proposed localization approach has been used on trench width down to $200 \mathrm{~nm}$ (see Fig. S4 of the supplementary material $^{30}$ ) and no fundamental limitations have as yet been encountered.

\section{Oxide removal and STM analysis of InP grown in trenches}

The HCl-based cleaning when combined with a low $\left(\sim 300^{\circ} \mathrm{C}\right)$ thermal annealing leads to, certainly on a blanket InP sample, a surface suitable for STM analysis and defect characterization. Targeting similar surface properties on $\mathrm{InP}$ grown in trenches, we have used the same optimized sample preparation procedure developed for blanket samples. A large scale analysis shows a morphological degradation of the $\{110\}$ sidewalls and a considerable anisotropic etching at the defect location (cf. Fig. S5 of the supplementary material ${ }^{30}$ ). According to van Dorp et al., an etch rate of $1.3 \mathrm{~nm} / \mathrm{min}$ is expected for a blanket $\{110\}$ surface using $2 \mathrm{M} \mathrm{HCl}^{35}$ However, the evaluated etch rate for our patterned sample is found to be $\sim 10$ times faster. The reason for such a discrepancy is at present unclear but could be linked to the higher defect density and to Fermi level pinning on the electrochemical etching. ${ }^{39}$ At a smaller scale, Fig. 7(a) shows an STM measurement carried out on top of a $500 \mathrm{~nm}$ wide trench after the chemical cleaning but prior to any thermal annealing. Two parallel lines, which are due to the presence of SFs/ twins, are identified. These planar defects lying in the $\{111\}$ plane create cuts perpendicular to the trench when reaching the top surface. Obviously, their topography is enhanced (defect decoration) by the anisotropy of the cleaning step. At a defect location, the etching process can either be stopped or vertically activated, hence modifying the topography. A sketch of these cuts is shown in Fig. 7(b). An equivalent structure was analyzed by transmission electron microscope (TEM) showing very clearly the presence of SFs/twins in the cross section image (Fig. 7(c)). The spatial distribution of these defects is comparable to what is observed in the STM image; i.e., they can be as close as $20 \mathrm{~nm}$ but also hundreds of $\mathrm{nm}$ apart. Figure 7(d) shows the sample after chemical cleaning and thermal annealing. The surface shows small atomically flat areas, almost equally distributed, among protrusions of the order of 3-5 nm high. Comparing InP grown in trenches with the blanket sample, the terrace size appears about one order of magnitude smaller. Although $\mathrm{HCl}$, in the concentration used here, is known to have a smoothing effect by removing atoms at the step edges and enlarging terraces, ${ }^{36}$ the small etch depth is obviously unable to induce a large smoothening effect. The equivalent etch for a blanket sample using $\mathrm{HCl}$ was found to be $\sim 0.5 \mathrm{~nm} .{ }^{35}$ Therefore, an initial
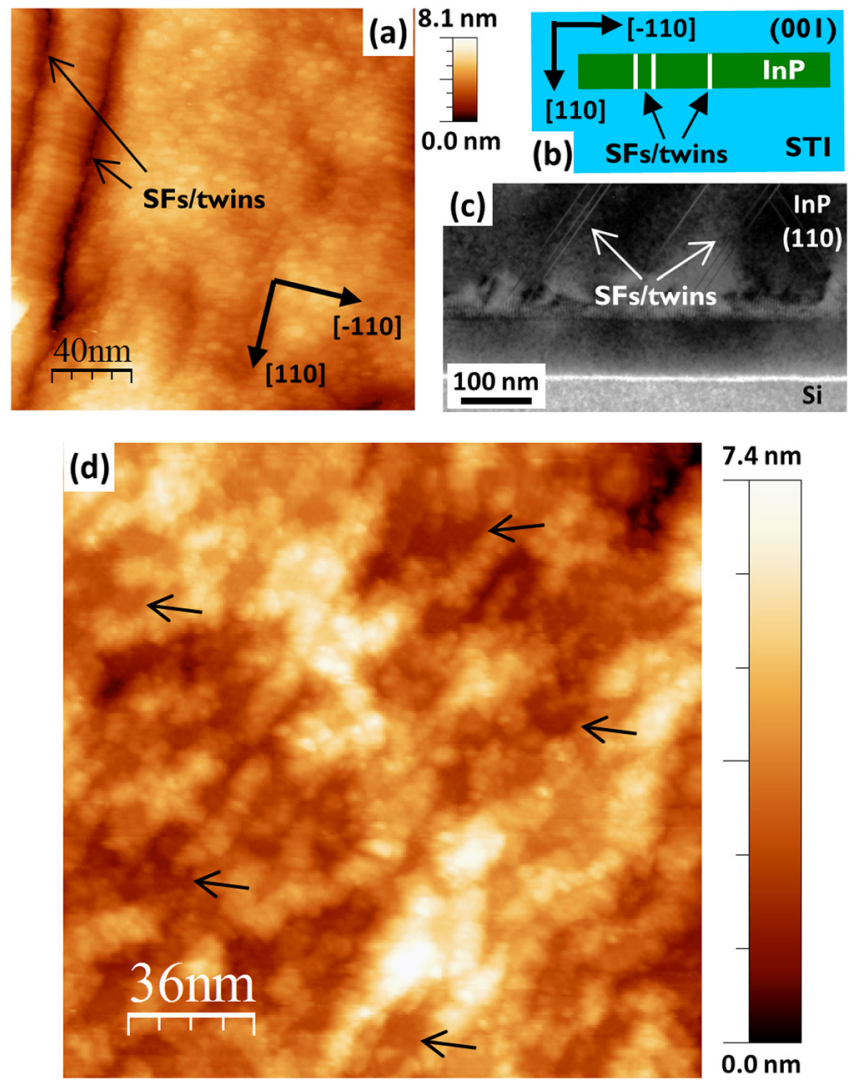

FIG. 7. (a) STM on a $500 \mathrm{~nm}$ trench after wet cleaning. The two lines, perpendicular to the trench, reveal the presence of SFs/twins reaching the top surface. The topography along the defect is enhanced by the wet cleaning step. Scan area $=200 \times 200 \mathrm{~nm}^{2}, \mathrm{~V}_{\mathrm{s}}=3.00 \mathrm{~V}, \mathrm{I}_{\text {set }}=150 \mathrm{pA}$. (b) Sketch of planar defects reaching the top surface and creating cuts. (c) TEM image of $\mathrm{InP}$ grown on a $\mathrm{Si}$ substrate which shows the presence of SFs and twins (cross section in parallel view). (d) STM on a $500 \mathrm{~nm}$ trench after wet cleaning and thermal annealing. The surface appears rough but with small atomically flat areas (some indicated by the arrows). Scan area $=180 \times 180 \mathrm{~nm}^{2}$, $\mathrm{V}_{\mathrm{s}}=-2.95 \mathrm{~V}, \mathrm{I}_{\mathrm{set}}=-130 \mathrm{pA}$.

roughness higher than a few nanometers becomes difficult to smooth. Moreover, the CMP step which the sample did undergo may also create cracks in the top atomic layers of the material affecting the terrace formation process. Larger atomically flat areas may be obtained by refining the $\mathrm{HCl}$ concentration and dip time. On the other hand, the anisotropy of the etching process needs to be taken into account since longer etching times can increase the surface roughness, especially at defect locations and at the $\{110\}$ sidewalls.

\section{CONCLUSIONS}

In this work, we have investigated different sample preparation and metrology approaches to enable STM surface and defect analysis of InP grown in narrow trenches. In order to reveal defects and measure accurately their properties, the native oxide must be removed and atomically flat surfaces need to be induced. It has been shown that a simple thermal treatment on InP cannot remove the native oxide without generating different surface modifications induced by the indium nucleation process. The effectiveness of HF and $\mathrm{HCl}$ based chemical cleanings has been investigated on blanket samples. The method based on $\mathrm{HCl}$ when combined 
with a low-temperature thermal annealing produces a better surface quality which is suitable for STM analysis and defect characterization. It allows to remove the native oxide layer, to avoid surface degradation, and to generate atomically flat terraces. Oxide-free InP surfaces were confirmed by STM analysis showing a $4 \times 2$ surface reconstruction.

For patterned samples, it has been identified that a higher defect density, roughness due to process steps (e.g., CMP), and the presence of different surface planes have a strong impact in the terrace formation process. Moreover, we have successfully performed STM analysis on InP trenches embedded in an oxide matrix using a combination of nc-AFM and STM. The chosen approach uses the same conductive AFM tip to perform nc-AFM to localize the trench and then to perform STM in the selected trench area. A proper selection of the AFM probe allows to obtain sub-nm resolution in STM mode. With this combined approach, trenches were easily identified and an STM surface analysis could be performed. Observation of surface reconstruction and stacking faults/ twins defects reaching the top surface has been shown.

\section{ACKNOWLEDGMENTS}

The authors would like to thank the MCA-SA Team for the TEM analysis and Dr. Richard Morris for his valuable comments and suggestions through the writing of this manuscript.

${ }^{1}$ J. A. del Alamo, Nature 479, 317 (2011).

${ }^{2}$ D. Cohen and C. B. Carter, J. Microsc. 208, 84 (2002).

${ }^{3}$ E. A. Fitzgerald and N. Chand, J. Electron. Mater. 20, 839 (1991).

${ }^{4}$ C. Merckling, N. Waldron, S. Jiang, W. Guo, O. Richard, B. Douhard, A. Moussa, D. Vanhaeren, H. Bender, N. Collaert, M. Heyns, A. Thean, M. Caymax, and W. Vandervorst, J. Appl. Phys. 114, 033708 (2013).

${ }^{5}$ G. Wang, M. R. Leys, R. Loo, O. Richard, and H. Bender, Appl. Phys. Lett. 97, 121913 (2010).

${ }^{6}$ M. Paladugu, C. Merckling, R. Loo, O. Richard, H. Bender, J. Dekoster, W. Vandervorst, M. Caymax, and M. Heyns, Cryst. Growth Des. 12, 4696 (2012).

${ }^{7}$ H. Zhao, Y. Chen, J. H. Yum, Y. Wang, F. Zhou, F. Xue, and J. C. Lee, Appl. Phys. Lett. 96, 102101 (2010).

${ }^{8}$ See http://www.itrs.net/ITRS\%201999-2014\%20Mtgs,\%20Presentations\% 20\&\%20Links/2013ITRS/2013Chapters/2013Metrology_Summary.pdf for International Technology Roadmap for Semiconductors, Metrology Summary, Accessed 8 September 2015, 2013.

${ }^{9}$ R. J. Hamers, R. M. Tromp, and J. E. Demuth, Phys. Rev. Lett. 56, 1972 (1986).

${ }^{10}$ R. M. Feenstra, Surf. Sci. 299/300, 965 (1994).

${ }^{11}$ F. J. Giessibl and B. M. Trafas, Rev. Sci. Instrum. 65, 1923 (1994).
${ }^{12}$ Z. Majzik, M. R. Tchalala, M. Švec, P. Hapala, H. Enriquez, A. Kara, A. J. Mayne, G. Dujardin, P. Jelínek, and H. Oughaddou, J. Phys. Condens. Matter 25, 225301 (2013).

${ }^{13}$ A. J. Weymouth, T. Wutscher, J. Welker, T. Hofmann, and F. J. Giessibl, Phys. Rev. Lett. 106, 226801 (2011).

${ }^{14}$ D. Sawada, Y. Sugimoto, K. Morita, M. Abe, and S. Morita, Appl. Phys. Lett. 94, 173117 (2009).

${ }^{15}$ M. Iwatsuki, K. Suzuki, S. Kitamura, and M. Kersker, Microsc. Microanal. 5, 208 (1999).

${ }^{16}$ F. Riesz, L. Dobos, and J. Karanyi, J. Vac. Sci. Technol. B 16, 2672 (1998).

${ }^{17}$ C. R. Bayliss and D. L. Kirk, J. Phys. D 9, 233 (1976).

${ }^{18}$ D. Kikuchi, Y. Matsui, and S. Adachi, J. Electrochem. Soc. 147, 1973 (2000).

${ }^{19}$ D. Kikuchi and S. Adachi, Mater. Sci. Eng. B 76, 133 (2000).

${ }^{20}$ T. Chass, H. Peisert, P. Streubel, and R. Szargan, Surf. Sci. 331/333, 434 (1995).

${ }^{21}$ Y. Sun, Z. Liu, F. Machuca, P. Pianetta, and W. E. Spicer, J. Appl. Phys. 97, 124902 (2005).

${ }^{22}$ E. Taglauer, Appl. Phys. A 51, 238 (1990).

${ }^{23}$ F. Stietz, T. Allinger, V. Polyakov, J. Woll, A. Goldmann, W. Erfurth, G. J. Lapeyre, and J. A. Schaefer, Appl. Surf. Sci. 104/105, 169 (1996).

${ }^{24}$ C. Merckling, N. Waldron, S. Jiang, W. Guo, N. Collaert, M. Caymax, E. Vancoille, K. Barla, A. Thean, M. Heyns, and W. Vandervorst, J. Appl. Phys. 115, 023710 (2014).

${ }^{25}$ I. Horcas, R. Fernandez, J. M. Gomez-Rodriguez, J. Colchero, J. GomezHerrero, and A. M. Baro, Rev. Sci. Instrum. 78, 013705 (2007).

${ }^{26}$ Q. Guo, M. E. Pemble, and E. M. Williams, Surf. Sci. 468, 92 (2000).

${ }^{27}$ Y. Ishikawa, T. Fukui, and H. Hasegawa, J. Vac. Sci. Technol. B 15, 1163 (1997).

${ }^{28}$ S. Ohkouchi and I. Tanaka, App. Phys. Lett. 59, 1588 (1991).

${ }^{29}$ O. Crisan, Trans. F: Nanotechnol. 17, 154 (2010).

${ }^{30}$ See supplementary material at http://dx.doi.org/10.1063/1.4936895 for InP surface morphology after a thermal anneal at $400^{\circ} \mathrm{C},\langle 110\rangle$ oriented terraces generated by a moving In cluster, methodology to enable STM analysis of trenches embedded in oxides, STM analysis of a $200 \mathrm{~nm}$ wide trench, and $\mathrm{HCl}$ impact on InP grown in trenches.

${ }^{31}$ M. Shimomura, N. Sanada, Y. Fukuda, and P. J. Moller, Surf. Sci. 359, L451 (1996).

${ }^{32}$ M. M. Sung, C. Kim, H. Bu, D. S. Karpuzov, and J. W. Rabalais, Surf. Sci. 322, 116 (1995).

${ }^{33}$ V. Chab, L. Pekarek, I. Ulrych, J. Suchy, K. C. Prince, M. Peloi, M. Evans, C. Comicioli, M. Zacchigna, and C. Crotti, Surf. Sci. 377-379, 261 (1997).

${ }^{34}$ D. Cuypers, S. De Gendt, S. Arnauts, K. Paulussen, and D. H. van Dorp, ECS J. Solid State Sci. Technol. 2, P185 (2013).

${ }^{35}$ D. H. van Dorp, D. Cuypers, S. Arnauts, A. Moussa, L. Rodriguez, and S. De Gendt, ECS J. Solid State Sci. Technol. 2, P190 (2013).

${ }^{36}$ D. Cuypers, D. H. van Dorp, M. Tallarida, S. Brizzi, T. Conard, L. N. J. Rodriguez, M. Mees, S. Arnauts, D. Schmeisser, C. Adelmann, and S. De Gendt, ECS J. Solid State Sci. Technol. 3, N3016 (2014).

${ }^{37}$ O. Ozcan and M. Sitti, Micro Nano Lett. 7, 329 (2012).

${ }^{38}$ J. L. Hutter and J. Bechhoefer, Rev. Sci. Instrum. 64, 1868 (1993).

${ }^{39}$ A. J. Bard, A. B. Bocarsly, F. F. Fan, E. G. Walton, and M. S. Wrighton, J. Am. Chem. Soc. 102, 3671 (1980). 